\title{
Health-related quality of life among hypertensive patients compared with general population norms.
}

\begin{abstract}
The 36-item short form (SF-36) health survey is the most popular generic HRQOL instrument that has been widely used to measure several population studies and variety of health conditions, including hypertension. The objectives of this study were to compare the HRQOL of hypertensive patients with that of a general population norm and analyze differences according to the presence of comorbid conditions such as diabetes mellitus and cardiovascular comorbidities. A total of 388 hypertensive patients was included in this crosssectional study. Socio-demographic information and the presence of diabetes mellitus and cardiovascular comorbidities were recorded. HRQOL was assessed by using SF-36 instrument. General linear models were used to identify statistically significant differences in scale scores. Hypertensive patients reported lower scores in six SF-36 dimensions except bodily pain and role emotional dimension when compared with Malaysian norms. After adjusting for socio-demographic variables (age, gender, education and employment), SF-36 scores in the presence of diabetes mellitus and cardiovascular comorbidities were comparably limited and both had statistically significantly lower scores than hypertensive patients without comorbidities. Hypertension reduced HRQOL and cardiovascular comorbidities exacerbate reductions. Hence, future research of effective interventions should include ensuring desirable HRQOL as well as controlling blood pressure to prevent or reduce comorbidities of hypertension.
\end{abstract}

Keyword: Health; Quality of life; Hypertension; Comorbidity; Health status; Malaysia. 